\title{
Factors influencing professional decision making on unplanned hospital admission:
}

\author{
a qualitative study
}

\begin{abstract}
Background

Unplanned admissions to hospital are a challenge for healthcare systems internationally. In the UK variation in unplanned admission rates across geographical areas, general practices and GPs remains largely unexplained.

\section{Aim}

To identify factors influencing professional decision making around unplanned hospital admission.

\section{Design and setting}

Qualitative study with a purposive sample of health and social care professionals from three primary care trusts, two acute hospitals, social services and an ambulance service in the South West of England.
\end{abstract}

\section{Method}

Semi-structured interviews were conducted with 19 professionals. Interviews were audio-recorded and transcribed. Data were analysed thematically drawing on the constant comparative method.

\section{Results}

The main factors influencing professional decision making around unplanned admissions were: lack of availability of seamless care on a 24/7 basis; 'professional tribalism' and poor information flow; service targets and performance management; commissioning culture and the impact of a 'market approach' and clinical governance structures, tolerance of risk and the role of peer support. A tension was perceived between the need to reduce unplanned admissions by tolerating more risk in primary care and a risk averse culture in secondary and emergency care.

\section{Conclusion}

Professional decision making that leads to unplanned admission to hospital is influenced by a range of organisational and individual health or social care professional factors. Finding ways to modify and ameliorate the effects of these systems and individual influences should be considered an important goal in the design of new interventions.

\section{Keywords}

decision making; emergency care; general practice; patient admission; qualitative research

\section{INTRODUCTION}

Unplanned or emergency admissions to hospital are expensive and can be distressing for patients and carers. However, despite efforts to reduce unplanned admissions, rates continue to increase, creating growing pressure on health system resources. ${ }^{2,3}$

In the UK patients can access emergency hospital care with or without referral from a GP. Thus examination of factors influencing hospital admission in the NHS may be relevant to countries with similar healthcare systems.

A number of interventions to reduce avoidable admissions have been introduced in the NHS, with limited success. ${ }^{4}$ Many of these interventions have not been informed by rigorous quantitative evidence regarding efficacy and cost-effectiveness, ${ }^{3}$ or by qualitative evidence on factors influencing professional decision making that may lead to admission.

There is some evidence to suggest that clinician factors play an important part in determining hospital admission rates. ${ }^{5}$ In a study exploring variations in GPs' out-ofhours emergency referrals, factors reported to influence referral behaviour included: risk management and individual tolerance of risk; access to alternative care; and time taken to identify and organise alternatives to admission. ${ }^{6}$ Decisions about referral for emergency admission may involve conflicts of interests for GPs, who have to strike a balance between concerns for the patient's health, consequences for other stakeholders and their own professional reputations.'
However, variation in referral rates remains poorly understood and largely unexplained. ${ }^{8}$

The aim of this study is to explore factors impacting on professional decision making around unplanned hospital admission across the primary, emergency, and social care sectors, in order to enhance the understanding of variations in rates of admission.

\section{METHOD}

The study took place in three primary care trusts (PCTs), two acute hospitals with emergency departments (ED or A\&E), an ambulance service and social services in one geographic area with a range of unplanned admission rates in England, UK. The ambulance service covered both hospitals, all three PCTs and social services providers. The other services were not necessarily coterminous.

\section{Sampling and data collection}

Purposeful sampling was used to select participants involved in decision making that may result in unplanned admissions. " The study sought to include individuals across primary, emergency, and social care sectors, with a variety of professional roles. Key roles to be included were identified in collaboration with the study advisory group and individuals fulfilling those roles were then approached via email with an invitation to participate.

Individual, indepth interviews were conducted in the participant's workplace. Interviews took the form of a guided conversation, using a flexible topic guide
RL Simmonds, BSc, MSc, research associate; A Shaw, BA, MSc, PhD, senior research fellow in primary care research; S Purdy, BSc, MPH, MD, FRCGP reader in primary care, the Centre for Academic Primary Care, School of Social and Community Medicine, University of Bristol, Bristol. Address for correspondence

Sarah Purdy, School of Social and Community Medicine, University of Bristol, Canynge Hall, 39
Whatley Road, Bristol, BS8 2PS, UK

E-mail: sarah.purdyabristol.ac.uk

Submitted: 6 February 2012; Editor's response: 13 March 2012; final acceptance: 24 April 2012. CBritish Journal of General Practice

This is the full-length article (published online 29 Oct 2012) of an abridged version published in print. Cite this article as: Br J Gen Pract 2012; DOI: 10.3399/bjgp12X658278 


\section{How this fits in}

Unplanned admissions to hospital are a challenge for healthcare systems and variation in unplanned admission rates across general practices remains largely unexplained. This study used qualitative methodology to identify factors influencing professional decision making around unplanned hospital admission. Health and social care professionals reported the following influences on decision making: lack of availability of seamless care on a 24/7 basis; 'professional tribalism' and poor information flow; conflicting service targets and performance management; commissioning culture and the impact of a 'market approach'; clinical governance structures, tolerance of risk and the role of peer support. By identifying factors that influence decision making across health and social care, these findings highlight areas that could be addressed by interventions to reduce unplanned hospital admissions.

to cover areas relevant to the research as well as allowing participants to raise new unanticipated issues. Interviews were digitally recorded and transcribed verbatim. Interviews took place from late 2010 to spring 2011.

\section{Data analysis}

Analysis began alongside data collection, with early analysis informing topics and perspectives to be pursued in an iterative process. Analysis was guided by the constant comparative method and began with detailed reading of each transcript, noting initial emerging issues. ${ }^{10}$ The qualitative software package NVivo 8 was used to aid the coding of transcripts, the development of codes into broader categories and themes, and the retrieval of data relevant to these themes. Open coding of individual transcripts generated an initial coding framework, which was added to and refined, with coded material regrouped and recoded as new data were gathered. ${ }^{10}$ The codes were built into broader categories and, through comparison across transcripts, higherlevel recurring themes were developed. Data within themes were scrutinised for disconfirming and confirming views across the range of participants. ${ }^{11}$ All authors regularly discussed the evolving coding framework, any apparently anomalous data, and the final themes to ensure the credibility and legitimacy of the findings. Emerging findings were verified with representatives of the relevant health and social care sectors who formed the study advisory group.

\section{RESULTS}

Nineteen health and social care professionals were interviewed: five from primary care, seven from secondary care and seven from community and emergency care (Table 1).

A range of factors impacting decision making that leads to unplanned hospital admissions emerged from participants' accounts. Commonalities in these factors across the professional groups interviewed reflected both systems issues and individual professionalfactors. Systemsissuesincluded a range of long-standing organisational features of primary, secondary and social care as well as more recent aspects of the current commissioning culture in the NHS.

\section{'Falling between the cracks': lack of} availability of $24 / 7$ care

The lack of availability of seamless care across healthcare interfaces, on a 24 hours a day, 7 days a week basis, was a recurring issue in participants' accounts regarding influences on decision making:

I think it is a 7-day week issue [demand for health care services] but I think the hospitals still work on the 5 days a week and most organisations work on 5 days a week and between about lunchtime on Friday and Monday morning nothing happens ... so people tend to end up in hospital sometimes whether they need it or not' (P11, social worker).

A lack of coherence in the provision of health care outside normal working hours was seen to result in patients 'falling between the cracks' in the system and being admitted to hospital perhaps unnecessarily.

A lack of service capacity due to poor weekend staffing levels was attributed as a major barrier to the provision of round-theclock care:

'Teams often run out of capacity on a Friday before a weekend ... and that's largely because it's difficult to employ people at weekends. '(P3, primary care)'

Participants reflected on the economics of increasing staff numbers to increase weekend functionality, but tended to conclude that this would not prove cost effective when weighed against any financial savings made through averting hospital admissions:

Using professional people that are only working weekends ... and then you end up having to pay a lot 
more money for those services and there isn't an endless amount of money ... and actually, quite frankly, once you get into that arena, it [24/7 care] stops being worthwhile.' (P3, primary care)

\section{Poor information flow and "professional tribalism'}

The lack of coherence between computerised patient information systems across different healthcare sectors and the variable quality of written information between professionals were repeatedly cited as factors influencing decision making:

Information flows remain very poor despite the plethora of IT systems that are out there ... if GPs wrote better referral letters, if consultants wrote better discharge letters and both of them talked in proper language to each other ... then the GP taking over the care would be able to actually make a better informed decision about the next steps and support that journey.' (P15, primary care)

Poor communication between health and social care was also cited by a number of participants, particularly in relation to accessing patient notes:

Researcher (R): "s there anything you can identify, in your job, you think well if only we could do this, that would make communication so much easier...?

\section{Table 1. Study participants}

\begin{tabular}{|c|c|c|}
\hline Staff based in primary care & Staff based in secondary care & $\begin{array}{l}\text { Staff based in community or } \\
\text { emergency care }\end{array}$ \\
\hline Out-of-hours GP & $\begin{array}{l}\text { Patient participation } \\
\text { Facilitator Acute Trust }\end{array}$ & $\begin{array}{l}\text { Risk assessor } \\
\text { Home Care Team, } \\
\text { Social Services }\end{array}$ \\
\hline GP and commissioner & $\begin{array}{l}\text { Team member } \\
\text { (admission avoidance, } \\
\text { multidisciplinary, primary care } \\
\text { team) Emergency Department, } \\
\text { Acute Trust }\end{array}$ & Community Matron \\
\hline Commissioner PCT & Matron/bed manager Acute Trust & $\begin{array}{l}\text { Ambulance Service response, } \\
\text { clinical staff member }\end{array}$ \\
\hline GP & $\begin{array}{l}\text { Team member, } \\
\text { GP Support Unit, } \\
\text { Emergency Department, } \\
\text { Acute Trust }\end{array}$ & $\begin{array}{l}\text { Team member, Single Point of } \\
\text { Access Triage Team }\end{array}$ \\
\hline \multirow[t]{3}{*}{ GP } & Hospital social worker & District nurse \\
\hline & $\begin{array}{l}\text { Senior clinician } \\
\text { Emergency Department, } \\
\text { Acute Trust }\end{array}$ & $\begin{array}{l}\text { Team member, Rapid } \\
\text { Response Team }\end{array}$ \\
\hline & $\begin{array}{l}\text { Senior nurse, Medical Admissions } \\
\text { Unit, Acute Trust }\end{array}$ & Community social worker \\
\hline
\end{tabular}

Participant (P): 'Yes, the computer systems really... sometimes you know I ring another ... [colleague] like the District Nurse and I've got to explain so much ... I just wish I could just email her all the case notes and she could just read them.' (P22, Social worker)

While the diversity of information technology systems was seen to be an important contributor, over and above this poor information flow was attributed to the presence of professional boundaries and 'tribalism' that hampered informed clinical decision making about patient care:

We have computer systems that share information, we've got computer systems that could share more information if we didn't have all the, professional tribalism that sits around it. It's not information governance ... it's about professional tribalism ... we could actually do more electronic information sharing if people gave up some of their kind of prejudices or stereotypes.' (P10, secondary care)

It also contributed to 'political' decision making around the funding of services and information systems:

P: '... I think it was a political thing really I think because we used to have the hub for [IT system] in our control room and it's gone from there now and I don't know if it costs a lot of money for the ambulance service to have it on their system, I don't know.'

R: 'Right. So you could look at patients' notes?'

P: 'Yes, I mean it wasn't ever there in the house [patient's home] because we were supposed to have laptops that allowed us to do it instantaneously but we never got those either even though there was funding for them. '(P14, emergency care)

More profoundly, there was the perception by some participants that only 'lip service was paid to the notion of patient-centred care in some areas of the health service. Service providers were often viewed as prioritising the needs of their service rather than patients, which could lead to poor information flow to other sectors:

'First of all, I think we pay lip service to the fact that the patient is at the centre of the journey. I don't believe that actually most professionals do put most patients at the centre of the journey. They put their service at the centre of what they're doing for the patient.'(P15, primary care) 
This was especially relevant for patients with long-term conditions who are more likely to receive care from health and social care services.

\section{Service targets and performance management}

Service delivery targets and performance management also emerged as interconnected factors impacting professional decision making about unplanned hospital admissions. For example: despite the introduction of highly trained emergency care practitioners (ECPs) to the ambulance service to avert avoidable admissions, the introduction of response time targets to monitor service performance resulted in ECPs being pulled out' of admission avoidance calls to meet response time targets:

'They [Ambulance Service Trust] have recently increased pressure on US ... if there's a red call [8-minute response time] ... they will interrupt us, take us out of that job [avoidable admissions] and send us to the red call ... That's just purely to do with wanting somebody there within 8 minutes. It's nothing to do with quality of care ... so their remit hasn't really been hospital avoidance. '(P14, emergency care)

The nationally implemented 4-hour waiting time target in ED was viewed as influencing professional decision making in favour of a hospital admission. Participants explained how this 4-hour performance target can drive risk-averse decision making in favour of admission, particularly for more junior staff. Rather than allowing patients to wait to be seen in the ED and risk failing to meet this performance target, clinicians were perceived as often opting for admission even if this is not necessarily in the patient's best interests:

'Most A\& E doctors, again, want to do what's best for the patient but the junior staff are probably more risk averse ... more senior staff are probably more likely to be able to turn people round, but you have the 4-hour target which is a big driver to move someone from an A\&E Department into a formal bed to trigger an admission ... so that 4-hour target will tend to drive people into admission. '(P5, primary care)

'They [A\&E] had a 4-hour target and if somebody is sat there it is quicker to put them in a bed so they meet their target, rather than wait until somebody gets involved and turns them around.' (P17, intermediate carel

\section{Commissioning culture and the impact of a 'market' approach}

Participants' accounts of how the commissioning culture impacts on professional decision making emphasised the ways in which the 'micro politics' of funding produced incentives and disincentives to hospital admission. Despite healthcare policy to locate more services in the community, admission avoidance, and discharge interventions were reported to lack capacity. It was suggested that PCTs were paying 'lip service' to the provision of community-based health care.

Broader economic and macro political drivers, such as the introduction of a 'market' approach to commissioning and the privatisation of services, were additionally felt to impact professional decision making and the quality of patient care. For example, a hospital-based participant explained how the funding of emergency care via the NHS fee for service payment mechanism - 'payment-by-results' (PbR) - was perceived to incentivise hospital admissions while augmenting adversarial relationships between secondary care and the PCT:

'Because of the PbR system there's an incentive to admit people because you get paid more money. So you could imagine the hospitals are going out onto the streets with a big net and getting people and dragging them in and saying you know you must come into this hospital and we'll charge the commissioning body, the PCT for your admission.' (P21, secondary care)

The same participant also explained how financial incentives may influence clinician decision making in primary care if a 'market approach' is adopted by the new GP led commissioning consortia:

'The incentivisation for GPs is a bit, for my money it's a bit too close to their personal profits I think ... The commissioning consortia, it's very clear, it's gonna be a major lever for them but that, of course, just drives down quality because they say well you know we'll cut this, we'll get rid of that, we'll move this, you know we'll get a cheaper version ... It will incentivise people for the wrong reasons.' (P21, secondary care)

GPs' decisions regarding hospital admission may end up being influenced primarily by financial drivers to provide the cheapest treatment option rather than by the ethic of providing the best quality patient care. 
Some concerns were expressed that services already in place to help avert hospital admissions were under-resourced. There was a perception that a degree of rhetoric existed at senior levels within the healthcare system regarding the value of interventions to avoid admissions, that was not supported by appropriate levels of funding to successfully implement such services. Failures in systems supposed to facilitate care for patients in the community, resulting from under-resourcing of such services, may leave clinicians with little choice but to refer to hospital while discouraging them from accessing these services in the future:

The difficulty is that the Single Point of Access [SPA] line is, is manned 24/7 but the services that they filter through are either full to capacity because they're understaffed, staff sickness, maternity leave, job vacancies. So what's happened is they've put a lot of staff to man that SPA line but the other teams that, who are there to help... safely discharge patients and care for people in their own home ... aren't able to take on [the work] because of staffing issues. What's happening is that at a much senior level what's being promised is not what's being delivered.' (P2, secondary care)

\section{Clinical governance structures, tolerance of risk, and the role of peer support}

Participants described how clinical governance structures within the health service shaped their approach to risk, including the degree to which they feel able to tolerate risk within their decision making about potential hospital admission.

A tension between avoiding risk and averting hospital admissions was perceived, particularlyamong primary care participants. A 'double think' was seen to exist within the healthcare system, with large secondary or emergency care organisations adopting top down systems to wrap themselves up in processes to completely avoid risk', while the more autonomous working culture of primary care encouraged individual primary care clinicians to tolerate and manage more risk to avert hospital admissions. A primary care participant argued that GPs were more able to tolerate risk due to being able to individually 'own', and take responsibility for, their decisions. Thus GPs were perceived by some secondary care professionals to be the key players in preventing unplanned hospital admissions:

We've got a little bit of confusion really about whether we think being risk averse is a good or bad thing. Often we talk about 'that's a risk averse type of service' as being a negative thing in the context of urgent care, because we want people not to go into hospital ... larger and larger organisations involving clinical staff ... will wrap themselves up in processes which completely avoid risk ... If you're a clinical governance lead or the medical director of a service you don't want to be taking risks. You don't want to be responsible for a lot of people on the ground that you don't necessarily know. So inevitably larger and larger organisations involving clinical staff, who are not necessarily doctors, will wrap themselves up in processes which completely avoid risk ... GPs are key here because they're often making decisions and the key reason why they're able to be less risk averse ... they are actually able to own their own decisions. So they are able to take direct responsibility as an individual for the decision that they make ...' (P3 primary care)

Having access to peer support in decision making was identified as an important factor in helping professionals to appropriately manage risk, and potentially avert an avoidable hospital admission:

You are making some decisions ... [and] yes it is quite a lonely process and it's really important to feel that you've got a peer or a parent type figure to ask or refer to.' (P9, primary care)

While this was particularly noted by professionals working in primary care, participants across the health and social care sectors emphasised the value of a supportive inter-professional working ethos for robust decision making. Peer support was seen to be fostered in an organisational ethos that was patient centred, multiprofessional and team based.

\section{DISCUSSION}

\section{Summary}

Health and social care professionals reported the following influences on decision making that may lead to an unplanned hospital admission: lack of availability of seamless care on a 24/7 basis; professional tribalism' and poor information flow; service targets and performance management; commissioning culture and the impact of a 'market approach'; and clinical governance structures, tolerance of risk and the role of peer support.

\section{Strengths and limitations}

To the authors' knowledge, this is the first qualitative study on decision making 
regarding unplanned admissions that has included a wide range of health and social service staff. Sampling across service sectors allowed to access a broad range of perspectives on inter- and intra-service issues that can impact on professional decision making

As only a few participants were recruited from each service sector some of the findings may represent idiosyncratic views from a specific perspective. As the study sampled to maximise variation in the professional groups represented, there was less scope for fine grained exploration of factors influencing decision making on unplanned admissions within each professional group. As rural areas report lower rates of emergency care use the inclusion of such a locality would have presented the opportunity to explore this factor.,12 The study was undertaken in one geographic area and some issues identified, such as access to patient record systems, may not be a problem in other areas.

\section{Comparison with existing literature}

Previous studies have reported that GPs experience conflicts of interest in decision making about emergency admissions., This study supports these findings, and adds a multiprofessional perspective by identifying a variety of potentially conflicting interests and rationalities across different health and social care sectors. These areas of tension may impact professional decision making and contribute to unplanned hospital admissions.

For example, professional tribalism and rivalries between services may inhibit the flow of patient information and the provision of seamless health care on a $24 / 7$ basis, favouring the needs of the service over the needs of the patient. ${ }^{13,14}$ Conflicting rationalities with regard to the management of risk may impact professional decision making with regard to unplanned admissions. While secondary and emergency care clinical governance frameworks are designed to minimise risk, GPs are being encouraged to tolerate risk in decision making. However, a risk tolerant approach to averting unplanned admissions in primary care may become mired by a lack of capacity in community based services to support the avoidance of an admission, or by a risk adverse culture in other services.

Haddow et al ${ }^{14}$ explored the issue of organisational identity in the implementation of a new, nationally integrated telephone advice and consultation service (NHS 24), concluding that seamless, inter-professional working across traditional organisational boundaries, requires recognition of the complex ownerships, and identities that exist within different parts of the health service..$^{15}$ Similarly, the professionals in this study identified how complexity has resulted in a lack of coherence across and within services, which impacts on decision making and promotes unplanned admission.

The presence of inter-organisational politics between hospitals, commissioners and primary care was raised by participants as an issue impacting decision making regarding hospital admission. Under $\mathrm{PbR}$, PCTs have been incentivised to prevent admissions as the full national tariff, or fee, is retained by the PCT for each admission avoided. ${ }^{16}$ However, there is evidence to suggest that $\mathrm{PbR}$ can induce hospitals to 'game' the system to their financial advantage for example, by accepting clinically inappropriate admissions from ED departments. ${ }^{17}$ Accounts from participants indicate perceptions about 'gaming' the system are present within professional groups across the healthcare sectors. It will be interesting to see how the new clinical commissioning consortia being introduced in the NHS will impact decision making regarding unplanned admission and whether further conflicts of interest will be experienced by GPs and their colleagues in other health and social care sectors.

\section{Implications for future research}

This study makes some contribution to understanding variations in admission rates. Future research developing interventions that address the problems highlighted around risk management across services, communication and IT, and 24-hour care provision, especially in patients with multimorbidities or complex needs, would provide solutions for overcoming these issues.

this study. We would also like to thank our advisory group for their encouragement, expert advice and enthusiasm. We thank the National Institute for Health Research for inclusion in their portfolio of studies.

\section{Discuss this article}

Contribute and read comments about this article on the Discussion Forum: http://www.rcgp.org.uk/bjgp-discuss 


\section{REFERENCES}

1. Henderson C, Sheaff R, Dickinson A et al. Unplanned hospital admissions of older people: the impact of governance. Final report. NIHR Service Delivery and Organisation programme, 2011.

2. Oster A, Bindman A. Emergency department visits for ambulatory care sensitive conditions: insights into preventable hospitalizations. Med Care 2003; 41(2): 198-207.

3. Purdy S. Avoiding hospital admissions. What does the research evidence say? London: The King's Fund. http:// mww.kingsfund.org.uk/publications/avoiding_ hospital.html (accessed 6 Aug 2012).

4. Blunt I, Bardsley M, Dixon J. Trends in emergency admissions in England 20042009: is greater efficiency breeding inefficiency? London: Nuffield Trust, 2010. http:// www.nuffieldtrust.org.uk/publications (accessed 6 Aug 2012).

5. Rossdale M, Kemple T, Payne S, et al. An observational study of variation in GPs' out-of-hours emergency referrals. Br J Gen Pract 2007; 57(535): 152-154.

6. Calnan M, Payne S, Kemple T, et al. A qualitative study exploring variations in general practitioners out-of-hours referrals to hospital. Br J Gen Pract 2007; 57(542): 706-713.

7. Dempsey O, Becker H. Heads you win, tails I lose: a critical incident study of GPs' decisions about emergency admission referrals. Fam Pract 2002; 19(6): 611-616.

8. O'Donnell C. Variation in GP referral rates: what can we learn from the literature? Fam Pract 2000; 17(6): 462-471.

9. Patton $\mathrm{MQ}$ : Qualitative research and evaluation methods. 3rd edn. London: Sage, 2002

10. Strauss AL, Corbin J. Basics of qualitative research: grounded theory procedures and techniques. 2nd edn. London: Sage, 1998.

11. Pope C, Ziebland S, Mays N. Qualitative research in health care: analysing qualitative data. BMJ 2000; 320(7227): 114-116.

12. Campbell N, Iversen $L$, Farmer J, et al. A qualitative study in rural and urban areas on whether--and how--to consult during routine and out of hours. BMC Fam Pract 2006; 7: 26.

13. Richardson S, Asthana S. Inter-agency information sharing in health and social care services: the role of professional culture. Brit J Soc Work 2006; 36(4): $657-669$

14. Haddow G, O'Donnell CA, Heaney D. Stakeholder perspectives on new ways of delivering unscheduled health care: the role of ownership and organizational identity. J Eval Clin Pract 2007; 13(2): 179-185.

15. Salisbury C, Bell D. Access to urgent health care. Emerg Med J 2010; 27(3): 186-188.

16. Mannion R, Street A. Managing activity and expenditure in the new NHS market. Public Money Manage 2009; 29(1): 27-34.

17. Ellis R. Creaming, skimping and dumping: provider competition on the intensive and extensive margins. J Health Econ 1998; 17(5): 537-555. 